\section{Molecular radiotherapy}

John Buscombe, consultant in nuclear medicine, Department of Nuclear Medicine, Addenbrooke's Hospital, Cambridge

Shaunak Navalkissoor, consultant in nuclear medicine, Department of Nuclear Medicine, Royal Free Hospital, London

Over the past 10 years the term 'molecular imaging' has become increasing familiar, and often (mis)used as a code for positron emission tomography (PET). In some senses this is correct as PET exploits increased uptake of a molecule designed to target a specific tissue whether by a physiological process or secondary to pathology.

Nuclear medicine was originally conceived as a therapeutic specialty, not primarily for imaging. The role of molecular radiotherapy has changed since the first patients were exposed to therapeutic unsealed sources of radioisotopes 70-80 years ago, using simple isotopes such as radiophosphorous (P-32) or radioiodine (I-131). Since then, complex treatments have been developed such as radioimmunotherapy for non-Hodgkin's lymphoma (NHL) or Y-90 selective internal radiotherapy (SIRT) for liver metastasis.

Treatment with P-32 and I-131 are still available, although the use of the former for polycythaemia is now limited. I-131 remains widely used to treat both benign and malignant thyroid disease and has become routine for the former. They have the advantage of being inexpensive, require a relatively straightforward infrastructure for safe delivery and are administered regularly in many UK centres. Other treatments such as radiation synovectomy or radiopeptide therapy of neuroendocrine tumours (NETs) require specific expertise and are less widely available.

There is good evidence that molecular radiotherapies are well tolerated, associated with limited side effects and can improve both quality of life and survival.

The recent development of other radionuclide therapies has led to increased cost, requiring dedicated inpatient facilities and demanding specialist skills. A recent review of molecular radiotherapy across the UK showed that whilst the administration of I-131 is fairly widespread, the use of new therapeutic radiopharmaceuticals is limited primarily to a few centres in the SouthEast and North-West of England. ${ }^{1}$ Access to treatment is, therefore, constrained both by drug availability and by the small number of specialists with the appropriate knowledge and experience to undertake this type of therapy.

\section{Benign disease treatment}

\section{Radioiodine}

Although often considered as a treatment for cancer, radionuclide therapies are frequently used to manage benign disease. The most common therapeutic application in the UK is radioiodine (I-131) treatment of hyperthyroidism and non-toxic goitre. I-131 is highly effective, safe and probably the most cost-efficient available treatment for hyperthyroidism. ${ }^{2-4}$ Some patients and referrers may be concerned about potential adverse effects of radioactivity, but these reservations can usually be dealt with by appropriate discussion and education. A lack of knowledge and inertia from referrers would seem to present the more serious impediments to increasing the number of patients treated.

\section{Radiation synovectomy}

Radiation synovectomy is commonly performed in Germany, but offered by only a few centres in the UK. It can provide important, durable benefit in patients with significant mono-arthropathies. Treatment is administered by intra-articular radiopharmaceutical injection, usually under radiological or ultrasonographic guidance. $^{5-7}$ Several different radionuclide agents are available, the choice governed by the size of the joint being treated. Lowenergy beta particle-emitting agents are used for the small joints of the hands and feet, for example, whereas high-energy, long range beta-emitting isotopes are better suited to large joints such as the knee. Radiosynovectomy is particularly effective in reducing pain and increasing movement in haemophilia-related post-haemorrhage synovitis of the knees and ankles. ${ }^{8}$

\section{From pain relief to extending life}

Radiotherapy is an important treatment option for metastatic bone pain palliation. Local field external beam radiotherapy is highly effective in patients with relatively limited skeletal metastases. Disseminated, painful bone metastases following hormone or chemotherapy failure can be better managed using hemibody radiation or systemic bone-seeking radionuclides. The advantages of the radionuclide approach are excellent, with selective bone targeting and low incidence of side effects.

Patients are selected on the basis of bone scintigraphy demonstrating increased uptake at sites of accelerated bone turnover in the bone surrounding the metastases. The target cell is not the tumour cell itself but the surrounding fibroblasts and osteoblasts which produce the pain mediators such as substance $\mathrm{P}$ and slow releasing substance. ${ }^{9}$

\section{Radionuclides for pain relief}

The different radionuclides used for bone pain palliation offer distinct physical properties such as half-life duration and emitted radioactivity path length, which in turn influence clinical response.

Samarium-153 (Sm-153) lexidronate, for example, has a relatively short half-life and less penetrating beta particle emission than the major alternative, the calcium analogue strontium-89 (Sr-89). Sm-153 lexidronate treatment relieves pain rapidly and rarely leads to significant myelosuppression, although repeated injections at three or four month intervals may be required for sustained benefit. By comparison, pain relief may be delayed for two to four weeks after Sr-89 administration. Bone marrow recovery may be slow, but symptom response duration is prolonged and may exceed six months following a single injection. ${ }^{10-12}$

These treatments can be co-administered with other systemic treatments for bone pain such as bisphosphonates or chemotherapy, ${ }^{13,14}$ although the latter is associated with a higher incidence of bone marrow suppression. 


\section{Radium-223}

A new approach involves the use of low activity, bone-seeking alpha-emitting radionuclides given repeatedly at four-week intervals to allow bone marrow recovery. In phase II clinical trials, radium-223 (Ra223) improved symptoms, reduced bone alkaline phosphatase and increased overall survival compared with placebo. ${ }^{15}$ A phase III trial of Ra-223 versus placebo in over 900 men with prostate cancer reported a 14-month median overall survival in the Ra-223 treated group compared with 11 months on placebo. ${ }^{16}$

\section{Networks to treat neuroendocrine tumours}

An expanding role of molecular radiotherapy has involved the palliative treatment of patients with disseminated NETs. Despite their low incidence, these tumours progress slowly such that the prevalence of NETs among patients attending a gastrointestinal tumour clinic approaches $10 \% .{ }^{17}$ Many of these tumours are hypersecretory, leading to intractable symptoms such as the carcinoid syndrome. Despite this slow growth NETs paradoxically often present late with disseminated disease that cannot be managed surgically and are typically chemotherapy resistant. ${ }^{17}$

\section{I-131 mIBG}

The first radiopharmaceutical agent used to treat NETs was I-131 metaiodobenzylguanidine (I-131mIBG), a catecholamine analogue with similar properties to noradrenaline. It has been most successfully used in neuroblastoma, malignant phaeochromocytoma $^{18,19}$ and NETs. ${ }^{20,21}$ There are no randomised control trials (RCTs) using I-131mIBG but case control studies show reduced symptoms and survival improved by at least two years in patients treated with I-131mIBG compared with untreated patients. ${ }^{22}$

One of the advantages of molecular radiotherapy in NETs is that the rate of success can be predicted by pre-imaging. There is positive uptake on diagnostic I-123 or I-131mIBG scans in $50-80 \%$ of patients with NET and nearly $90 \%$ have uptake of radiolabelled somatostatin analogues which attach to the type 2 receptor subtype (Fig 1).

\section{Octreotide derivatives}

Many academic institutions have developed therapeutic radiopeptides using modified octreotide derivatives. The most promising, octreotate, can be linked via a chelator to beta particle-emitting isotopes such as lutetium-177 (Lu-177) or yttrium-90 (Y-90) for use in metastatic NETs. The results in over 1,500 patients, reported by a number of centres around Europe, consistently show disease stability/overall response in about $70 \%$ of patients ${ }^{23-38}$ (Table 1). Disease stabilisation appears to translate to prolonged median overall survival which may extend beyond five years. Most centres observed that patients who fail to show significant radiological response immediately after therapy may demonstrate a late response, with tumour shrinkage up to one year after the last treatment. ${ }^{35}$

To date, the promise of this treatment approach has been restricted by the paucity of RCT data. A multicentre phase III study of Lu-177-labelled DOTATATE is planned in 2012-2013 which, it is hoped, will provide the infrastructure and evidence base to support future use.

\section{Lymphoma}

Follicular NHL is a radiosensitive disease and presents an ideal target for molecular radiotherapy. The chimeric monoclonal antibody rituximab has antitumour activity via targeting of the CD20 antigen expressed on mature lymphocytes and B cell tumours. This epitope is not present on precursor cells in the bone marrow so, after clearance of CD20 cells (including tumour sites) immunocompetent lymphocytes repopulate rapidly. ${ }^{39-40}$

Anti-CD20 antibodies have been labelled with I-131 (I-131 tositumomab) and Y-90 (ibritumomab tiuxetan). The latter is available in Europe and has been shown to double the response rate to rituximab. Treatment is administered by intravenous infusion on an outpatient basis and is well tolerated. $^{41,42}$ Y-90 ibritumomab tiuxetan consolidation treatment following induction chemotherapy prolonged progressionfree survival by a factor of three in patients with advanced follicular NHL. ${ }^{43}$ It has been included safely in conditioning regimens for autologous bone marrow transplantation in aggressive NHL, in combination with myeloablative BEAM (BCNU, etoposide, cytarabine and melphalan) chemotherapy. ${ }^{44}$

\section{The double whammy in liver tumours}

There is increasing use of Y-90 labelled particulates such as Y-90 resin beads (Y-90 SIR-Spheres $\left.{ }^{\circledR}\right)$ or glass balls (Y-90 TheraSpheres $\left.{ }^{\circledR}\right)$. This approach, sometimes called SIRT, combines selective partial
Table 1. Some results of treating disseminated neuroendocrine tumours with radiopeptides. ${ }^{23-38}$

\begin{tabular}{|c|c|c|c|c|c|}
\hline \multirow[b]{2}{*}{ Ref. } & \multirow[b]{2}{*}{ No. } & \multirow[b]{2}{*}{ Isotope } & \multicolumn{3}{|c|}{ Response } \\
\hline & & & $\begin{array}{c}\text { Symptomatic } \\
\text { (\%) }\end{array}$ & $\begin{array}{c}\text { Radiological } \\
\text { (\%) }\end{array}$ & $\begin{array}{l}\text { Median survival } \\
\text { (months) }\end{array}$ \\
\hline 25 & 87 & Y-90 & NR & 20 & NR \\
\hline 26 & 1,109 & Y-90 & 30 & 34 & 24 \\
\hline 27 & 58 & Y-90 & 58 & 9 & 37 \\
\hline 28 & 116 & Y-90 & 83 & 27 & NR \\
\hline 33 & 90 & Y-90 & 88 & 5 & 27 \\
\hline 35 & 57 & Y-90 & 72 & 23 & 22 \\
\hline 37 & 310 & Lu-177 & NR & 30 & 46 \\
\hline 38 & 51 & Lu-177 & NR & 33 & 40 \\
\hline
\end{tabular}


a

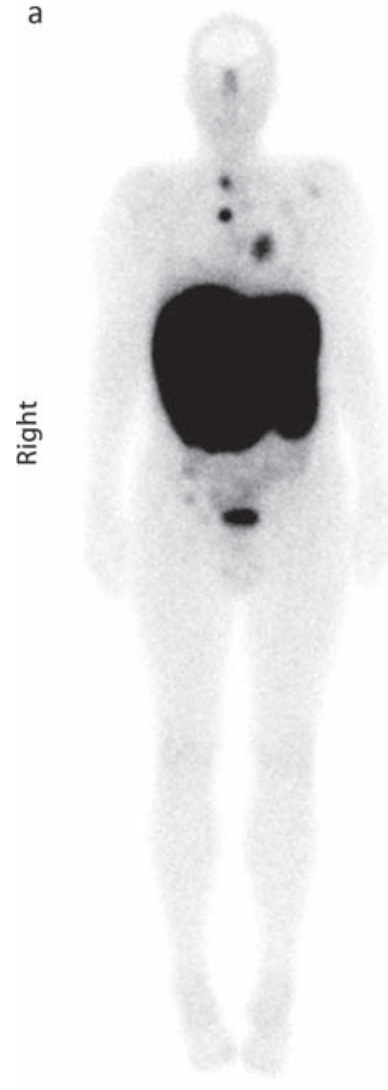

Anterior
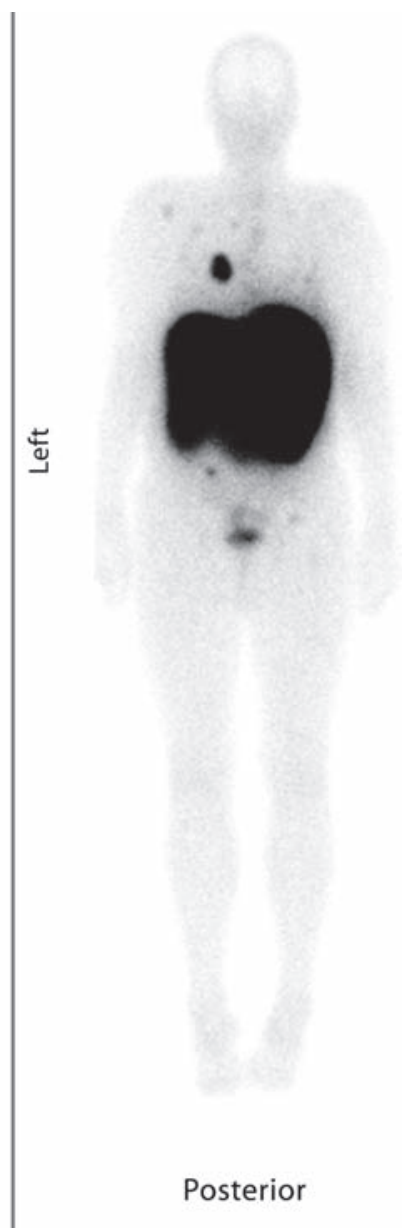

b

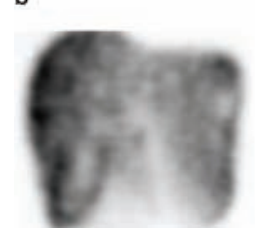

c
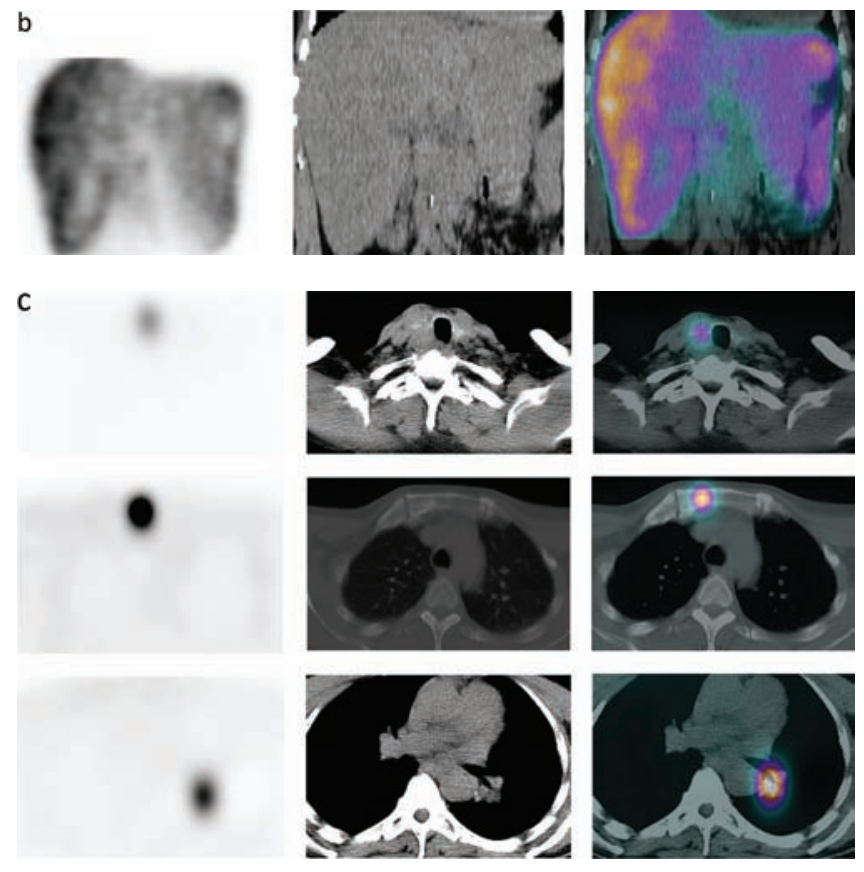

Fig 1. 60-year-old man with metastatic medullary thyroid cancer shown on a recent $\mathrm{CT}$ scan to have progressive disease. He was referred for peptide receptor radionuclide therapy (PRRT) having had a positive In-111-pentetretotide study, and was treated with four cycles of $7.4 \mathrm{GBq}$ of Lu-177 DOTATATE. Post-therapeutic imaging was performed the day after treatment with images demonstrating the biodistribution after the first cycle of treatment: (a) whole body study, (b) SPECT/ CT demonstrating good uptake in the liver metastases and (c) SPECT/CT demonstrating good uptake in the thyroid, left hilar mass and bone metastases in the sternum. 
embolisation with radiation to treat primary liver tumours or organ-confined metastases. Treatment delivery requires a team approach involving nuclear medicine, interventional radiology and oncology. In early studies SIRT was shown to lengthen progression-free survival from seven months to 12 months compared with chemotherapy alone in patients with metastatic colorectal cancer and liver predominant metastases. ${ }^{45}$ Cirrhosis does not preclude treatment, and Y-90 TheraSpheres in particular may be used safely in patients who have partial portal vein thrombosis. Early side effects include hepatic pain, typically occurring within 24-48 hours.

Several trials using radioembolisation to treat primary hepatocellular carcinoma (HCC $)^{46,47}$ report that it appears superior to transarterial chemoembolisation in downstaging patients with HCC. ${ }^{48,49}$ SIRT is increasingly used to treat patients with hepatic metastases secondary to colorectal and NETs. ${ }^{45,50}$ Further randomised trials are investigating a multimodality approach combining newer chemotherapy regimens with and without SIRT in metastatic colon cancer and HCC.

\section{Patient tolerability}

Molecular radiotherapy such as I-131, Y-90 anti-CD 20 antibodies or SIRT is generally given as a once only treatment. Some therapieslike I-131 mIBG and Lu-177 DOTATATE are fractionated at approximately 12-week time intervals both to reduce the likelihood of significant bone marrow toxicity and to deliver sustained benefit. For radiation protection reasons, high activity I-131-based treatments usually necessitate admission to hospital for one to five days (due to the high energy gamma rays) but many of the newer treatments using pure beta and alpha emitters can be administered safely as outpatient procedures.

I-131 treatment for benign thyroid disease may cause a transient sore throat but is otherwise well tolerated in patients rendered biochemically euthyroid prior to treatment. The likelihood of post-treatment hypothyroidism reflects both the underlying aetiology and administered activity. I-131 mIBG or Lu-177 DOTATATE may cause nausea for 24-48 hours and fatigue for a week post-treatment and transient myelosuppression.

Molecular radiotherapy is generally well tolerated. Common reported side effects such as nausea and myelosuppression are typically less severe and of shorter duration than would be expected following systemic chemotherapy. When formally tested as part of a trial, patients gave much higher scores for tolerability of molecular radiotherapy than of the alternatives. ${ }^{12,15,16,41,42,51}$

\section{Cost}

There is a broad spectrum of cost for molecular radiotherapy. Long established treatments such as I-131 for thyroid disease are inexpensive whereas costs continue to escalate for more recently developed radiopharmaceuticals. Some of the latter may be provided in the UK via the Cancer New Drugs Fund, but significant regional disparities persist and limit access to high quality treatment.

\section{Conclusions}

A detailed review of current research is beyond the scope of this review. However, it has been possible to show that molecular radiotherapy has multiple applications to treat benign and malignant diseases (Table 2). The field is expanding

\section{Table 2. Current available radionuclide therapies.}

\begin{tabular}{ll} 
Radiopharmaceutical & Clinical indication \\
I-131 & $\begin{array}{l}\text { Hyperthyroidism } \\
\text { Thyroid cancer }\end{array}$ \\
P-32 & Polycythaemia rubra vera \\
Sr-89 & Pain relief of bone metastases \\
Sm-153 lexidronate & Pain relief of bone metastases \\
Ra-223 & Improved survival in patients with bone \\
& metastases \\
Y-90, Re-186, Er-169 colloids & Radiation synovectomy \\
I-131 mIBG & Neuroblastoma \\
& Phaeochromocytoma \\
Y-90 ibritumomab tiuxetan & NETs \\
Y-90 particulates & Non-Hodgkin's lymphoma \\
Y-90/Lu-177 PRRT & Primary and secondary tumours in liver \\
\hline mIBG = metaiodobenzylguanidine; NET = neuroendocrine tumour; PRRT = peptide receptor radionuclide \\
therapy.
\end{tabular}

\section{Key points}

Molecular radiotherapy can be used in benign and malignant disease

Access to molecular radiotherapy varies across the UK

The treatment methods used involve simple molecules such as $131 \mathrm{I}$ sodium iodide to radiolabelled antibodies.

Neuroendocrine tumours respond to molecular radiotherapy when chemotherapy and biotherapy fail

With the arrival of radium treatment in metastatic prostate cancer molecular radiotherapy moves from palliating pain to improving survival

KEYWORDS: radionuclide, therapy, thyroid, lymphoma, neuroendocrine tumours, prostate cancer 
rapidly in parallel with advances in molecular imaging. Future developments are likely to include both new radiopharmaceuticals and exploitation of synergies between radionuclide and other cytotoxic therapies within multimodality treatment regimens.

\section{References}

1 Flux G, Moss L, Buscombe J et al. Molecular Radiotherapy in the UK: Current Status and Recommendations for Further Investigations. BIR Report 23. London: British Institute for Radiology, 2011.

2 Alimanovic-Alagic R, Kucukalic-Selimovic E, Mekic M. Efficiency and safety of radioactive iodine I-131 in treatment of thyroid disease. Med Arh 2009;63:295-6.

3 Sarkar SD. Benign thyroid disease: what is the role of nuclear medicine? Review. Semin Nucl Med 2006;36:185-93.

4 Patel NN, Abraham P, Buscombe J, Vanderpump MP. The cost effectiveness of treatment modalities for thyrotoxicosis in a U.K. center. Thyroid 2006;16:593-8.

5 Göbel D, Gratz S, von Rothkirch T et al. Radiosynoviorthesis with rhenium-186 in rheumatoid arthritis: a prospective study of three treatment regimens. Rheumatol Int 1997;17:105-8.

6 Göbel D, Gratz S, von Rothkirch T, Becker W. Chronic polyarthritis and radiosynoviorthesis: a prospective, controlled study of injection therapy with erbium 169 and rhenium. $186 Z$ Rheumatol 1997;56: 207-13. [In German]

7 O’Duffy EK, Clunie GP, Lui D et al. Double blind glucocorticoid controlled trial of samarium-153 particulate hydroxyapatite radiation synovectomy for chronic knee synovitis. Ann Rheum Dis 1999;58:554-8.

8 Calegaro JU, Machado J, DE Paula JC et al. Clinical evaluation after 1 year of 153-samarium hydroxyapatite synovectomy in patients with haemophilic arthropathy. Haemophilia 2009;15:240-6.

9 Mercadante S. Malignant bone pain: pathophysiology and treatment. Pain 1997;69:1-18.

10 Laing AH, Ackery DM, Bayly RJ et al. Strontium-89 chloride for pain palliation in prostatic skeletal malignancy. Br J Radiol 1991;64:816-22.

11 Dearnaley DP, Bayly RJ, A'Hern RP et al. Palliation of bone metastases in prostate cancer. Hemibody irradiation or strontium-89? Clin Oncol ( $R$ Coll Radiol) 1992;4:101-7.

12 Sartor O, Reid RH, Bushnell DL et al. Safety and efficacy of repeat administration of samarium Sm-153 lexidronam to patients with metastatic bone pain. Cancer 2007;109:637-43.
13 Storto G, Klain M, Paone G et al. Combined therapy of Sr-89 and zoledronic acid in patients with painful bone metastases. Bone 2006;39:35-41.

14 Sciuto R, Festa A, Rea S et al. Effects of low-dose cisplatin on 89 Sr therapy for painful bone metastases from prostate cancer: a randomized clinical trial. J Nucl Med 2002;43:79-86.

15 Nilsson S, Franzén L, Parker C et al. Bonetargeted radium-223 in symptomatic, hormone-refractory prostate cancer: a randomised, multicentre, placebo-controlled phase II study. Lancet Oncol 2007;8:587-94.

16 Parker C, Heirich D, O'Sullivan JM et al. Overall survival benefit of radium-223 chloride (Alpharadrin) in the treatment of patients with symptomatic bone metastases in castration-resistant prostate cancer (CRPC): a phase III randomized trial. Eur J Cancer 2011;47:3(suppl 2).

17 Caplin ME, Buscombe JR, Hilson AJ et al. Carcinoid tumour. Review. Lancet 1998;352:799-805.

18 Howard JP, Maris JM, Kersun LS et al. Tumor response and toxicity with multiple infusions of high dose 131I-MIBG for refractory neuroblastoma. Pediatr Blood Cancer 2005;44:232-9.

19 Gonias S, Goldsby R, Matthay KK et al. Phase II study of high-dose [131I] metaiodobenzylguanidine therapy for patients with metastatic pheochromocytoma and paraganglioma. J Clin Oncol 2009;27:4162-8.

20 Navalkissoor S, Alhashimi DM, Quigley AM et al. Efficacy of using a standard activity of (131)I-MIBG therapy in patients with disseminated neuroendocrine tumours. Eur J Nucl Med Mol Imaging 2010;37:904-12.

21 Safford SD, Coleman RE, Gockerman JP et al. Iodine-131 metaiodobenzylguanidine treatment for metastatic carcinoid. Results in 98 patients. Cancer 2004;101:1987-93.

22 Sywak MS, Pasieka JL, McEwan A et al. 131I-meta-iodobenzylguanidine in the management of metastatic midgut carcinoid tumors. World J Surg 2004;28:115762.

23 Chinol M, Bodei L, Cremonesi M, Paganelli $\mathrm{G}$. Receptor-mediated radiotherapy with Y-DOTA-DPhe-Tyr-octreotide: the experience of the European Institute of Oncology Group. Semin Nucl Med 2002;32:141-7.

24 Bodei L, Cremonesi M, Zoboli S et al. Receptor-mediated radionuclide therapy with 90Y-DOTATOC in association with amino acid infusion: a phase I study. Eur J Nucl Med Mol Imaging 2003;30:207-16.

25 Paganelli G, Bodei L, Handkiewicz Junak D et al. 90Y-DOTA-D-Phe1-Try3-octreotide in therapy of neuroendocrine malignancies. Biopolymers 2002;66:393-8.

26 Imhof A, Brunner P, Marincek $\mathrm{N}$ et al. Response, survival, and long-term toxicity after therapy with the radiolabeled somatostatin analogue [90Y-DOTA]-TOC in metastasized neuroendocrine cancers. $J$ Clin Oncol 2011;29:2416-23.

27 Valkema R, Pauwels S, Kvols LK et al. Survival and response after peptide receptor radionuclide therapy with [90Y-DOTA0,Tyr3] octreotide in patients with advanced gastroenteropancreatic neuroendocrine tumors. Semin Nucl Med 2006;36:147-56.

28 Forrer F, Waldherr C, Maecke HR, MuellerBrand J. Targeted radionuclide therapy with 90Y-DOTATOC in patients with neuroendocrine tumors. Anticancer Res 2006;26:703-7.

29 Waldherr C, Pless M, Maecke HR et al. Tumor response and clinical benefit in neuroendocrine tumors after 7.4 GBq (90) Y-DOTATOC. J Nucl Med 2002;43:610-6.

30 Bushnell D, O’Dorisio T, Menda Y et al. Evaluating the clinical effectiveness of $90 \mathrm{Y}-$ SMT 487 in patients with neuroendocrine tumors. J Nucl Med 2003;44:1556-6.

31 Bushnell D, Menda Y, Madsen $\mathrm{M}$ et al. Assessment of hepatic toxicity from treatment with 90Y-SMT 487

(OctreoTher(TM)) in patients with diffuse somatostatin receptor positive liver metastases. Cancer Biother Radiopharm 2003;18:581-8.

32 Gabriel M, Andergassen U, Putzer D et al. Individualized peptide-related-radionuclide-therapy concept using different radiolabelled somatostatin analogs in advanced cancer patients. Q J Nucl Med Mol Imaging 2010;54:92-9.

33 Bushnell DL Jr, O’Dorisio TM, O'Dorisio MS et al.90Y-edotreotide for metastatic carcinoid refractory to octreotide. J Clin Oncol 2010;28:1652-9.

34 Baum RP, Söldner J, Schmucking M, Niesen A. Intravenous and intra-arterial peptide receptorradionuclidetherapy (PRRT) using Y-90-DOTA-Tyr3-octreotate (DOTATATE) in patients with metastatic neuroendocrine tumours. Eur J Nucl Med 2004;44(Suppl):S238.

35 Cwikla JB, Sankowski A, Seklecka N et al. Efficacy of radionuclide treatment DOTATATE Y-90 in patients with progressive metastatic gastroenteropancreatic neuroendocrine carcinomas (GEP-NETs): a phase II study. Ann Oncol 2010;21:787-94.

36 Esser JP, Krenning EP, Teunissen JJ et al. Comparison of [(177Lu)-DOTA(0),Tyr3] octreotate and [(177Lu)-DOTA(0), Tyr3] octreotide: which peptide is preferable for PRRT? Eur J Nucl Med Mol Imaging 2006;33:1346-51.

37 Kwekkeboom DJ, de Herder WW, Kam BL et al. Treatment with the radiolabeled somatostatin analog [(177Lu)DOTA 0,Tyr3] octreotate: toxicity, efficacy, and survival. J Clin Oncol 2008;26: 2124-30. 
38 Bodei L, Cremonesi M, Grana CM et al. Peptide receptor radionuclide therapy with ${ }^{177}$ Lu-DOTATATE: the IEO phase I-II study. Eur J Nucl Med Mol Imaging 2011;38:2125-35.

39 Ryan DH, Nuccie BL, Abboud CN, Liesveld JL. Maturation-dependent adhesion of human B cell precursors to the bone marrow microenvironment. J Immunol 1990;145:477-84.

40 Maloney DG, Grillo-López AJ, White CA et al. IDEC-C2B8 (Rituximab) anti-CD20 monoclonal antibody therapy in patients with relapsed low-grade non-Hodgkin's lymphoma. Blood 1997;90:2188-95.

41 Vose JM, Wahl RL, Saleh M et al. Multicenter phase II study of iodine-131 tositumomab for chemotherapy-relapsed/ refractory low-grade and transformed lowgrade B-cell non-Hodgkin's lymphomas. J Clin Oncol 2000;18:1316-23.

42 Witzig TE, Gordon LI, Cabanillas F et al. Randomized controlled trial of yttrium-90labeled ibritumomab tiuxetan radioimmunotherapy versus rituximab immunotherapy for patients with relapsed or refractory low-grade, follicular, or transformed B-cell non-Hodgkin's lymphoma. $J$ Clin Oncol 2002;20:2453-63.

43 Morschhauser F, Radford J, Van Hoof A et al. Phase III trial of consolidation therapy with yttrium-90-ibritumomab tiuxetan compared with no additional therapy after first remission in advanced follicular lymphoma. J Clin Oncol 2008;26:5156-64.

44 Shimoni A, Avivi I, Rowe JM et al. A randomized study comparing yttrium-90 ibritumomab tiuxetan (Zevalin) and high-dose BEAM chemotherapy versus BEAM alone as the conditioning regimen before autologous stem cell transplantation in patients with aggressive lymphoma. Cancer 2012 Jan17. [Epub ahead of print].

45 Gray B, Van Hazel G, Hope M et al. Randomised trial of SIR-Spheres plus chemotherapy vs. chemotherapy alone for treating patients with liver metastases from primary large bowel cancer. Ann Oncol 2001;12:1711-20.

46 Gulec SA, Mesoloras G, Dezarn WA et al. Safety and efficacy of Y-90 microsphere treatment in patients with primary and metastatic liver cancer: the tumor selectivity of the treatment as a function of tumor to liver flow ratio. J Transl Med 2007;5:15.

47 Hilgard P, Hamami M, Fouly AE et al. Radioembolization with yttrium-90 glass microspheres in hepatocellular carcinoma: European experience on safety and long-term survival. Hepatology 2010;52:1741-9.

48 Riaz A, Lewandowski JR, Ryu RK et al. Chemoembolization vs radioembolization: comparison of toxicity, imaging response and long-term outcomes in 100 TACE vs 104 Y 90 patients. SIR (Society of Interventional Radiology), Annual Scientific Meeting 2009. J Vasc Interv Radiol 2009;20:S83.

49 Lewandowski RJ, Kulik LM, Riaz A et al. A comparative analysis of transarterial downstaging for hepatocellular carcinoma: chemoembolization versus radioembolization. Am J Transplant 2009;9:1920-8.

50 Chandra P, Yarandi SS, Khazai N et al. Management of intractable hypoglycemia with Yttrium-90 radioembolization in a patient with malignant insulinoma. Am J Med Sci 2010;340:414-7.

51 Bhattacharya S, Novell JR, Dusheiko GM et al. Epirubicin-Lipiodol chemotherapy versus 131 iodine-Lipiodol radiotherapy in the treatment of unresectable hepatocellular carcinoma. Cancer 1995;76:2202-10.

Address: Dr John Buscombe, Nuclear Medicine, Box 170, Addenbrooke's Hospital, Hills Road, Cambridge CB2 0QQ.

Email: john.buscombe@addenbrookes. nhs.uk

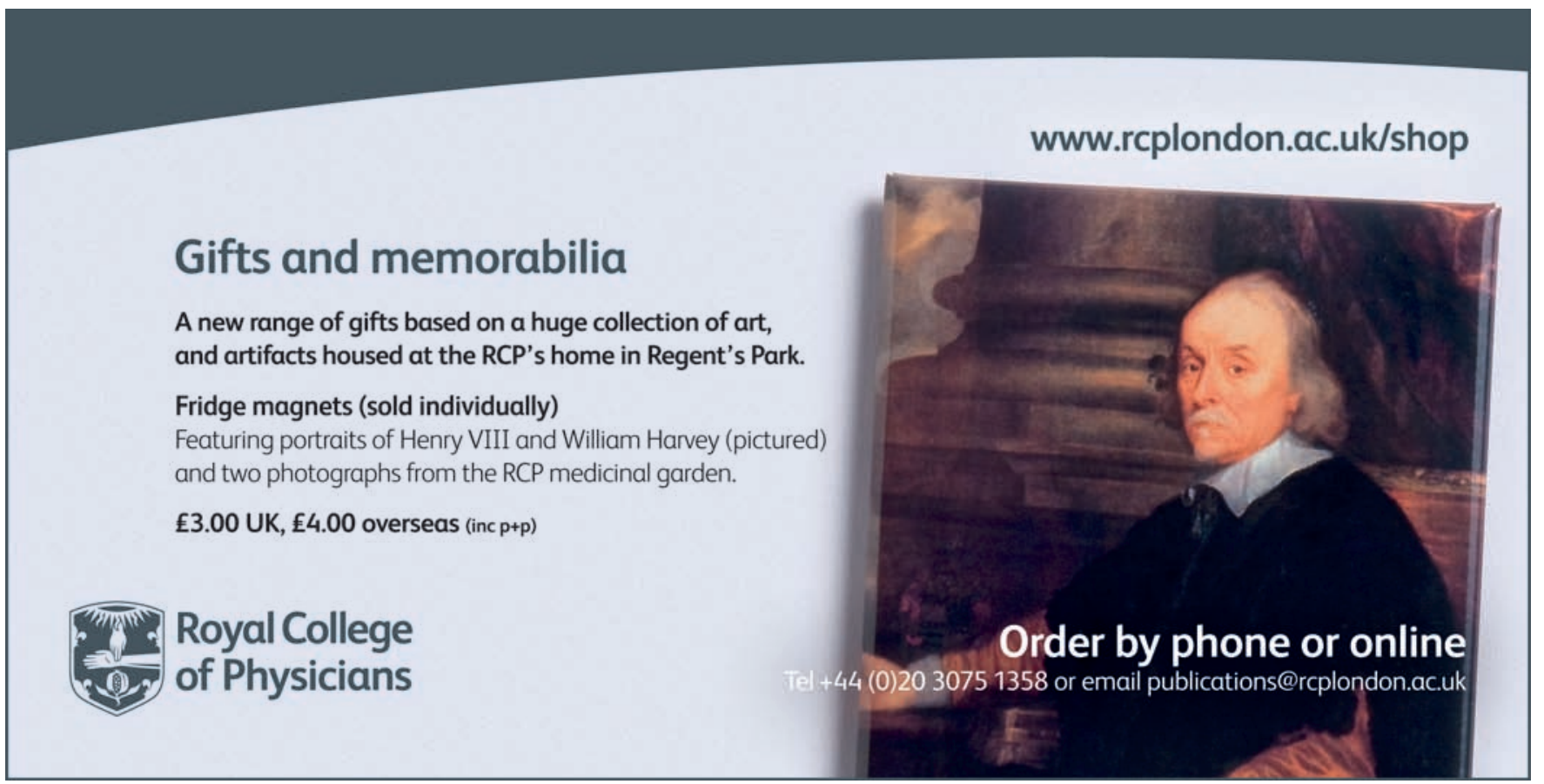

